\title{
Smoking and smoking cessation in pregnancy. Synthesis of a systematic review.
}

Grangé $G^{*}$ gilles.grange@aphp.fr, Berlin I, Bretelle F, Bertholdt C, Berveiller P, Blanc J, DiGuisto C, Dochez V, Garabedian C, Guerby P, Le Lous M, Perdriolle-Galet E, Peyronnet V, Rault $\mathrm{E}$, Torchin $\mathrm{H}$

Grangé G, Maternité Port-Royal, université de Paris, AP-HP.Centre, FHU Prema, 75014 Paris, France.

Berlin I, Département de pharmacologie, AP-HP, 75000 Paris, France; Centre universitaire de médecine générale et de santé publique, 1000 Lausanne, Suisse.

Bretelle F, Department of obstetrics and gynecology, conception hospital, Aix Marseille université, prenatal diagnosis Timone Conception, IHU, IRD, Assistance publique des Hôpitaux de Marseille (AP-HM), 13005 Marseille, France

Bertholdt C, Pôle de la femme, maternité régionale du CHRU de Nancy, 10, rue DrHeydenreich, 54000 Nancy, France; IADI, Inserm U1254, rue du Morvan, 54500 Vandœuvrelès-Nancy, France

Berveiller P, Service de gynécologie-obstétrique, CHI Poissy-St-Germain-en-Laye, 78300 Poissy, France

Blanc J, Service de gynécologie obstétrique, hôpital Nord, AP-HM, chemin des Bourrely, 13015 Marseille, France; EA3279, CEReSS, health service research and quality of life center, université Aix-Marseille, 13284 Marseille, France

DiGuisto C, Université de Paris, epidemiology and statistics research center/CRESS, Inserm (U1153 - Obstetrical, Perinatal and Pediatric Epidemiology Research Team (EPOPé)), INRA, hôpital Tenon, bâtiment Recherche, rue de la Chine, 75020 Paris, France; Maternité Olympe de Gouges, centre hospitalier régional universitaire Tours, Tours, France; Université François-Rabelais, 37000 Tours, France

Dochez V, Service de gynécologie-obstétrique, CHU de Nantes, 44093 Nantes, France. Garabedian C, EA 4489 - Perinatal Environment and Health, université de Lille, hôpital Jeanne-de-Flandre, clinique d'obstétrique, CHU de Lille, 59000 Lille, France.

Guerby P, Service de gynécologie obstétrique, hôpital Paule-de-Viguier, CHU de Toulouse, 330, avenue de Grande-Bretagne, 31059 Toulouse, France.

Koch A, Service de gynécologie-obstétrique, CHU de Strasbourg, 67000 Strasbourg, France.

Le Lous $M$, Département de gynécologie obstétrique et médecine de la reproduction, CHU de Rennes, Rennes, France; LTSI-Inserm, université de Rennes 1, UMR 1099, 35000 Rennes, France.

Perdriolle-Galet E, Pôle de la femme, maternité régionale du CHRU de Nancy, 10, rue Dr-Heydenreich, 54000 Nancy, France.

Peyronnet V, Service de gynécologie obstétrique, hôpital L.-Mourier, AP-HP, 92700 Colombes, France.

Rault E, Hôpital Femme-Mère-Enfant, 69500 Bron, France.

Torchin $\mathrm{H}$, Service de médecine et réanimation néonatales de Port-Royal, AP-HP, 75014 Paris, France; Université de Paris, epidemiology and statistics research center/CRESS, Inserm, INRA, 75004 Paris, France. 
Legendre G. CESP-Inserm, U1018, Team 7, Genre, Sexual and Reproductive Health, Paris-Sud University, Paris-Saclay University, UVSQ, Inserm, 94800 Villejuif, France; Department of obstetrics and gynecology, Angers university hospital, 49000 Angers, France.

\section{*Corresponding author:}

Gilles Grangé :

\section{Highlights}

- "Counselling", including all types of non-pharmacological interventions, has a moderate benefit on smoking cessation, birth weight and prematurity.

- Nicotine replacement therapies (NRT) may be prescribed to pregnant women who have failed to stop smoking after trying non-pharmacological therapies.

- In cases of temporary relapse or resumption of smoking, it should be recommended to continue with the NRT.

- It is not recommended to let the smoking status interfere with the choice of how to feed the new-born infant.

\section{Abstract}

\section{Objectives}

To provide up-to-date evidence-based guidelines for the management of smoking cessation during pregnancy and the post-partum period.

Study Design A systematic review of the international literature was undertaken between January 2003 and April 2019. MEDLINE, EMBASE databases and the Cochrane library were searched for a range of predefined key words. All relevant reports in English and French were classified according to their level of evidence ranging from 1 (highest) to 4 (lowest). The strength of each recommendation was classified according to the Haute Autorite de Santé (French National Authority for Health) ranging from A (highest) to C (lowest).

\section{Results}

"Counselling", including all types of non-pharmacological interventions, has a moderate benefit on smoking cessation, birth weight and prematurity. The systematic use of measuring expired air $\mathrm{CO}$ concentration does not influence smoking abstinence, however, it may be useful in assessing tobacco consumption prior to and after quitting.

The use of self-help therapies and health education are recommended in helping pregnant smokers quit and should be advised by healthcare professionals

Nicotine replacement therapies (NRT) may be prescribed to pregnant women who have failed to stop smoking after trying non-pharmacological interventions. Different modes of delivery and dosages can be used in optimizing their efficacy.

Smoking in the postpartum period is essential to consider. The same treatment options as during pregnancy can be used.

\section{Conclusion}

Smoking during pregnancy concerns more than a hundred thousand women each year in France resulting in a major public health burden. Healthcare professionals should be mobilised to employ a range of methods to reduce or even eradicate it.

Keywords: smoking during pregnancy; pre-and post-natal effects; smoking cessation during pregnancy 


\section{Introduction}

Smoking during pregnancy and the postpartum period continues to be a major health concern worldwide and in particular in France. To provide guidance for healthcare professionals involved in the clinical management of women during pregnancy and the postpartum period, the French College of Obstetricians and Gynaecologists (CNGOF) and the Francophone Society of Tobacco Addiction (Société Francophone de Tabacologie, SFT) produced this Expert Report and Recommendations. The expert group ran a systematic literature review identifying relevant articles through until mid-2019 by searching the MEDLINE, EMBASE databases and the Cochrane library.

The Expert Report and Recommendations are designed to be informative. A healthcare professional (midwife, obstetrician-gynaecologist, paediatrician, anaesthetist or any other healthcare professional) should have free choice to decide whether or not to apply any of the expert recommendations if they believe that the recommendation is not the most appropriate course of action for their patient. This applies especially for recommendations not supported by a high level of evidence.

The following four guidelines were applied to attribute the level of evidence i.e. the strength of associations to the research findings:

1. The working group used verbs in the present tense when the level of evidence (LE) is high (LE 1 and 2, and exceptionally LE3 when all the studies are in agreement on the outcome). In contrast when the level of evidence is found to be LE3 and 4 the verbs are used in the conditional tense.

2. Two results derived from the same study may produce different ratings (for example LE2 for one and LE3 for another outcome). This may occur if one of the outcomes was the main focus of the study and the other was a secondary outcome, or if the statistical significance of the study was considered insufficient for one of the two outcomes studied.

3. The scientific quality of an outcome is evaluated by the level of evidence (LE) [1], while the strength of the recommendation is classified by a grading system (A: highest, B, C: lowest) proposed by the Haute Autorité de Santé (HAS) (French National Authority for Health) [2].

4. Finally, a standardised sentence structure was employed to facilitate understanding of the recommendations:

a) 'it is recommended to use....' implies that the literature demonstrates that the intervention (verbal, behavioural, organisational, medical etc) improved the outcome and/or reduced the risks associated with smoking.

b) 'it is not recommended to use....' implies that the literature has not demonstrated that the intervention has therapeutic efficacy, or that it does not reduce the risks associated with smoking. It is therefore recommended not to use this intervention. It does not imply that its use is contra-indicated if the healthcare professional judges that the benefit to the individual outweighs the risk.

c) 'it is recommended not to use....' implies that the literature has demonstrated that the use of the intervention increases the risks which outweigh the benefits or that there are no benefits. It is therefore recommended to avoid using this intervention. 
This article intends to summarise the main findings and recommendations and refers to the original detailed systematic reviews in French.

\section{Epidemiology, risk factors, optimal time for smoking cessation and predictors of failure to stop [3].}

The prevalence of tobacco smoking during pregnancy throughout the world is estimated at $1.7 \%$ (95\% $\mathrm{Cl} 0.0-4.5 \%$ ) (LE3). In France, the prevalence of active smoking is approximately $30 \%$ pre-conception, $20-24 \%$ in the first trimester and $14-20 \%$ in the third trimester of pregnancy (LE3). There is great disparity according to different regions in mainland France $(9.4-24 \%)$ and its overseas territories (5.2\%) (LE3).

Risk factors include young age, low level of education, primiparity, geographical area in mainland France, the absence of a profession, a partner who smokes, the absence of a partner, and suffering from depression. Multiparity is a predictive factor for the failure to stop smoking during pregnancy (LE2).

\section{Physiological changes influencing the smoking behaviour of pregnant women. Assessment of smoking behaviour[4].}

The physiological changes that occur during pregnancy result in an increased metabolic and renal clearance of nicotine which can lead to a relative decrease in plasma or tissue-specific nicotine levels. This subsequently may increase withdrawal symptoms and desire to smoke. If a pregnant woman decides to stop smoking these physiological data should be considered in order to optimize nicotine replacement therapies by adapting the dosage to meet the woman's needs.

Methods of assessing smoke exposure include self-report questionnaires and objective measurements, for example, expired air carbon monoxide. The questionnaires used to evaluate tobacco exposure and addiction which are most frequently cited in the literature are the Fagerström Test for Cigarette Dependence (FTCD) and its two-question variant the Heaviness of Smoking Index (HSI). This latter questionnaire has the same or even better metric properties than the FTCD. Both closely correlate with plasma, salivary or urine concentrations of cotinine, the main metabolite of nicotine. There is insufficient evidence of their usefulness in predicting the rate of smoking during pregnancy. .

Due to the simplicity of their use, the FTCD or the HSI could be an additional tool to establish a treatment plan to enable pregnant smokers to stop smoking. The diagnostic criteria of DSM-V (Diagnostic and Statistical Manual of Mental Disorders version V) [5] could 
be used to assess tobacco addiction/tobacco use disorder in pregnant smokers, however, there are no specific studies assessing their specificity in pregnancy.

The measurement of expired air carbon monoxide (CO), end-product of tobacco combustion, is a simple tool that can be used throughout pregnancy and the post-partum period to identify women who smoke. there is no evidence documenting the role of this method helping smokers quit during pregnancy. Moreover, no study to date has shown any beneficial effect of expired air CO measurement on the prevention of obstetric complications associated with smoking.

The measurement of expired air $\mathrm{CO}$ is a simple non-invasive technique, leading to an immediate result at little cost. It could be helpful in demonstrating objectively the level of tobacco smoke exposure. This may create a starting point for further involvement in the smoking cessation process and provide a feedback for smoke exposure. Expired air CO testing has been shown to be beneficial as a screening method for smoking amongst pregnant women and help involve them into a smoking cessation program.

\section{Consequences of smoking during pregnancy. [6]}

Pregnant smokers are at increased risk of suffering a miscarriage or of having an ectopic pregnancy (LE2), with a dose-dependent toxic effect of tobacco consumption (LE2). Smoking during pregnancy is also a risk factor for a large number of congenital abnormalities (LE2) and is associated with an increased risk of placental abruption (LE2), placenta praevia (LE2), and intrauterine fetal death (LE2)(Table 1).

Regarding delivery and the postpartum period, smoking during pregnancy may be associated with an increased risk of delivery by Caesarean section, especially in women who smoke more than 10 cigarettes a day (LE3) and an increased risk of post-partum deep vein thrombosis (LE3).

Smoking in pregnancy is also associated with delivering a lower birth weight baby (LE2) and with an exposure/dose-dependent risk of preterm birth. The ban of smoking in public places is associated with a decrease in preterm birth rates (LE2).

\section{Smoking during pregnancy: impact on the child from birth to adulthood [7]}

Beyond pregnancy specific complications, smoking during pregnancy is also associated with negative health outcomes in the child ranging from physical complications through to psychological, behavioural and cognitive problems. However, it is difficult to disentangle the role of pre- and post-natal exposure, genetic, environmental and socio-economic factors.

Maternal smoking is known to be associated with sudden infant death syndrome (SIDS) (LE2). The different theories: pathophysiology, dose-dependent effect and the impact of stopping smoking, lean towards a causal relationship. 
Children born to mothers who smoked during pregnancy show reduced respiratory function from birth onwards when compared to those children born to non-smoking mothers (LE2). This effect persists into adult life. These children are at higher risk of lower respiratory tract infections in the first years of life and of asthma and respiratory symptoms throughout childhood (LE2).

Similarly, an association between maternal smoking during pregnancy and the risk of the child being overweight or obese has been demonstrated (LE2). Children born to smoking mothers may also at higher risk of central nervous system tumours (neuroblastomas) but not of leukaemias (LE3).

The effects of maternal smoking during pregnancy may have an impact beyond the first generation. Smoking during pregnancy probably has an impact on the future fertility of the child, and a trans-generational effect with an increased risk of asthma as seen in the grandchildren of smoking women (LE4). The pathophysiological mechanism are largely unknown, the most evoked are the epigenetic modifications.

Children born to mothers who smoke during their pregnancy are themselves more likely to smoke, to develop an addiction to nicotine and to start smoking at an early age (LE2).

Early analyses demonstrated a possible association between maternal smoking during pregnancy and intelligence quotient (IQ), learning disabilities (LE3), behavioural problems, especially attention deficit hyperactivity disorders (LE2) and academic performance (LE3).

\section{Consequences of passive smoking in pregnancy [8]}

The major risk factor associated with passive smoking is having a partner who smokes. Other risk factors may include sharing the home or car with a smoker, young age group (< $25 y$ rs old) and a low level of education (LE4).

Exposure to passive smoking appears to be highest at home or in the car with potential consequences to the pregnancy, most notably on the risk of prematurity (LE3). Passive smoking is also associated with an increased risk of intrauterine fetal death, fetal malformations, prematurity and birth weight lower than $2.5 \mathrm{kgs}$ (LE2).

Complete cessation of smoking at home could significantly reduces the risk of exposure to second-hand smoke compared to partial cessation (LE4).

It is recommended to advise women, as well as their families and close friends (particularly their partner) to create a smoke-free environment, notably at home and in the car (Professional Consensus).

Although no specific intervention can be recommended, a behavioural approach aimed at teaching the pregnant woman to negotiate with those around her may reduce her exposure and the effects of passive smoking on the pregnancy. 


\section{Other methods of tobacco consumption during pregnancy: electronic cigarette,} heated tobacco products, waterpipe and snus. [9]

Cigarettes are still the most commonly used tobacco product, however alternative tobacco and nicotine systems are also used widely. These include electronic cigarettes (vaping), heated tobacco products, waterpipes and snus (a smokeless, buccal absorption tobacco originating from Sweden). Healthcare professionals should be aware of these alternative tobacco/nicotine consumptions in order to comprehensively assess tobacco and nicotine use in pregnancy.

Nicotine exposure is maintained in users of nicotine-containing electronic cigarettes i.e. electronic nicotine delivery systems (ENDS). Even though the fetus is not exposed to the toxic combustible elements of the 'conventional' cigarette, research is necessary to assess the effects of the other components of electronic cigarettes, such as flavours, propylene glycerol, glycerol, volatile organic compounds, etc. and the overall benefit-risk ratio of using ENDS. As of today, the amount of data is insufficient to assess with confidence the health effects of alternative nicotine delivery systems, either in the general population or, more specifically, in pregnant women. Therefore, it is wise to follow the precautionary principle of do no harm. It is recommended to advise against starting smoking electronic cigarettes or continuing with these vaping products during pregnancy (Professional Consensus). Among women already using electronic cigarettes it is recommended to give the same advice that is given to smokers of cigarettes i.e. to stop vaping (Professional Consensus). At this current time there are no data on the use of $J u u l \circledast$ or assimilated products and heated tobacco in pregnant women. In the absence of such data, it is recommended to advise against the use of these products during pregnancy (Grade C). The use of well documented smoking cessation treatments with individual tailoring are suggested.

The smoking of a waterpipe has been found to have a negative impact on fetal growth. It is therefore recommended not to smoke a waterpipe during pregnancy (Grade A). The use of snus (oral tobacco originating from Sweden) should also be discouraged during pregnancy (Grade B).

\section{Public health policy regarding smoking during pregnancy [10]}

The establishment of a network of care services for pregnant women who smoke appears to be effective in aiding them to stop smoking provided that the pregnant women are helped in an ongoing, supportive manner notably by means of telephone calls, letters and other communication tools (LE3). Screening pregnant women for smoking is best achieved by systematically asking about cigarette and other tobacco use along with the measurement of expired air $\mathrm{CO}$ during pregnancy consultations.

Treatment options and individualised care should be discussed and reviewed regularly at follow up visits in collaboration with the network of local dedicated smoking cessation healthcare workers (Grade C). It is necessary to create local dedicated treatment options which are accessible to every pregnant smoker (Professional Consensus). 


\section{Non-pharmacological treatment options for smoking cessation during pregnancy [11]}

"Counselling" is a broad term used in studies which might include different types of advice (behavioural, motivational, supportive and therapeutic) delivered by a third party. It could be delivered on an individual or group basis, face to face or over the telephone.

Globally, "counselling" results in increased rates of smoking cessation by the end of the pregnancy (LE1) which is beneficial regarding birth weight and preterm birth (LE2). It is therefore recommended to offer counselling (simple advice and/or motivational counselling and/or cognitive behavioural therapy) to all pregnant women who smoke in order to encourage them to stop smoking (Grade A), however no single method has been found to be superior to another.

The acronym 5A's (ask, advise, assess, assist, arrange) summarises the brief interventions that each healthcare professional needs to employ when seeing a pregnant smoker.

Motivational counselling has been shown to be an effective tool in stopping smoking in the general population, but the same effect was not demonstrated among pregnant smokers. Analysis of behaviour modification and cognitive behavioural therapy do not demonstrate an advantage over usual treatment options offered to pregnant women (LE2).

The success of counselling techniques depends on the motivation of the smoker, her adherence to the treatment program, her overall monitoring and on the professional involved and the therapeutic relationship.

Feedback techniques (via expired air CO measurements, urinary cotinine levels as well as information on the potential negative effects of smoking delivered during fetal ultrasound scans) provide disappointing results, they do not increase smoking cessation rates. Their systematic use is not recommended (Grade C).

Financial incentives, particularly incentives based on rewarding abstinence from smoking, improve levels of smoking cessation by the end of the pregnancy and subsequently improve neonatal outcomes with a cost-benefit analysis in favour of their use (LE1). Further studies regarding the use of these methods and their cost-benefit analysis in France would allow recommendations of their use to be established.

Hypnotherapy and acupuncture have not been studied, or very little studied, in the pregnant smoking population.

Self-help methods (pamphlets, structured programs either written, via an application or a video delivered by telephone, smartphone or the internet) seem to be generally more effective than no intervention at all in the quest for the pregnant smoker to quit (LE2) but their benefit in addition to other treatment options has not been extensively studied. The use of self-help methods as a whole is recommended for the pregnant smoker in order to improve the rate of smoking cessation (Grade C). 
Health education is recommended, to include all treatment options with mention of their effectiveness (Grade B).

\section{Medical treatments for smoking cessation in pregnancy [12]}

Nicotine is the principle ingredient in tobacco that leads to addiction. Different types of nicotine replacement therapy (NRT) are available in France and throughout the world:

- NRT administered as short-acting buccal absorption products have a rapid onset of action allowing individuals to titrate their needs easily, giving them control over their tobacco cravings and withdrawal symptoms. Examples of these are nicotine gum, lozenges, inhalers and buccal sprays. The nasal spray is not available in France.

- Transdermal administration of nicotine, in the form of nicotine patches, ensures a continuous delivery over either 16 or 24 hours according to the pharmaceutical form prescribed.

The various pharmaceutical forms of NRT with differing doses can be combined and this improves the effectiveness, however, this remains to be studied in the pregnant population.

At the current time, there are no data regarding the use of NRT on the rate of smoking cessation in the preconception period.

Randomised control trials of NRT in pregnancy do not demonstrate a significant rate of smoking cessation during pregnancy or by the end of pregnancy (LE1). However, analysis of all available studies looking at the use of NRT during pregnancy does show that its use is associated with stopping smoking during or by the end of pregnancy (LE2)[13].

The prescription of NRT products during pregnancy should be aimed at alleviating the craving/desire to smoke and at alleviating withdrawal symptoms such as irritability, restlessness, anxiety, insomnia, depressive mood and increased appetite etc.

Prescribing NRT is not associated with an increased risk of pregnancy disorders (LE2) and no cause for concern has been reported in postmarkeing surveillance. The prescribing of NRT could be offered to all pregnant smokers who have failed to give up smoking on their own (Grade B). These products could be prescribed by the healthcare professional at the beginning of the pregnancy (Professional Consensus). It is however also recommended that the pregnant smoker is referred to a smoking cessation specialist in order to perform further assessment and to tailor the initial prescription to her needs (Professional Consensus). The data from the literature do not allow for a general recommendation regarding either the mode of delivery (fast-acting versus slow-acting pharmaceutical forms) or the optimal duration of the treatment (Professional Consensus).

Maintaining the use of NRTs in the event of a relapse may be associated with an overall reduction in tobacco consumption (LE3). This would suggest in recommending to continue with the NRT if a relapse occurs. (Professional Consensus). 
Due to a lack of data, it is not recommended to prescribe non-nicotine, second-line treatments, such as bupropion, varenicline (Grade $\mathrm{C}$ ), nortriptyline and clonidine (Professional Consensus), to pregnant women.

\section{Smoking and breastfeeding [14]}

Nicotine and its main metabolite, cotinine, are detectable in breastmilk. The maternal use of NRT whilst breastfeeding may reduce the rate of smoking or increase the rate of stopping smoking thus preventing the baby from being exposed to second-hand smoke and the harmful components it contains.

There are few experimental data about NRT during breastfeeding. However, post-marketing data show lack of risk using NRT whilst breastfeeding.

A smoke-free interval between smoking a cigarette and breastfeeding could result in a lower nicotine concentration in the breast milk exposing the child to less nicotine (LE4). Therefore, it is recommended not to smoke or not to use short-acting NRT immediately before breastfeeding i.e. to keep the interval between smoking or NRT and breastfeeding as long as possible (Professional Consensus).

There is an association between maternal smoking during the post-partum period and the risk of various childhood conditions including: childhood asthma and lower respiratory tract infections (LE2), childhood learning difficulties (LE3), infant colic (LE2), obesity (LE2) and sudden infant death syndrome (LE2). However, the protective effect of breastfeeding for these conditions has not yet been demonstrated.

It is not recommended to let the smoking status interfere with the choice of how to feed the new-born infant (Professional Consensus). Breastfeeding is associated with a reduction in tobacco consumption and/or stopping smoking (LE2). In order to limit tobacco consumption, it is recommended to promote breastfeeding amongst women who continue to smoke (Grade B).

\section{Post-partum: risk management to prevent relapse [15]}

Resumption of smoking in the post-partum period is associated with multiple factors, including quality of life, anxiety level, ability to manage stress and to return to a normal body weight (LE2), although there are insufficient data to suggest a conclusive relationship to any factor. The risk of resuming smoking in the post-partum period is high (up to $82 \%$ at 1 year in France). The main factors with continuing to abstain from smoking at this time are breastfeeding, not having a smoker at home, and the absence of symptoms of post-partum depression (LE2).

There are no data concerning the effectiveness of NRT in preventing a relapse to smoking in the post-partum period.

It is recommended to offer "counselling" to prevent smoking relapse during the postpartum period (Grade B). It is however not recommended to systematically offer cognitive 
behavioural therapy as this has not shown any benefit in preventing the resumption of smoking in the post-partum period (Grade B).

Financial incentives seem to be effective (LE3). Medico-economic data are necessary to evaluate their cost-benefit ratio. The use of information technologies to prevent the risk of relapse also remains to be studied.

\section{Conclusion}

The clinical management of pregnant women who smoke should involve a network of healthcare professionals to assess her smoking habits in a non-judgmental manner in order to provide her with support and pharmacological treatments to stop smoking. All healthcare professionals should be involved in this goal in a continuous manner throughout the pregnancy. The consultation with expert advisers in stopping smoking is an additional aid.

\section{Funding}

The work to elaborate this Expert Report and Recommendations was funded by a grant of Santé Publique France. None of the working group members were funded by this grant.

\section{Declaration of interest}

Authors declare having no financial and personal relationships with other people or organizations that could inappropriately influence (bias) this work.

\section{REFRENCES}

1. OCEBM Levels of Evidence Working Group*. The Oxford 2011 Levels of Evidence. Trans Durieux N, Pasleau F, Howick J. Oxford Centre for EvidenceBased Medicine. http://www.cebm.net/index.aspx?o=5653; (consulté le 07/04/2020)

2. Haute Autorité de Santé 2013. Niveau de preuve et gradation des recommandations de bonne pratique. https://www.hassante.fr/upload/docs/application/pdf/2013-

06/etat_des_lieux_niveau_preuve_gradation.pdf (consulté le 07/04/2020)

3. [Epidemiology and Risk Factors of Smoking During Pregnancy (Excluding Coaddictions) CNGOF-SFT Expert Report and Guidelines for Smoking Management during Pregnancy]. Dochez V, Diguisto C. Gynecol Obstet Fertil Senol. 2020 Apr 1:S2468-7189(20)30137-9. doi: 10.1016/j.gofs.2020.03.024.

4. [Physiological and Psychological Data influencing Pregnant Women Smoking Behavior CNGOF-SFT Expert Report and Guidelines for Smoking Management during Pregnancy]. Berveiller P, Rault E, Guerby P. Gynecol Obstet Fertil Senol. 2020 Apr 1:S24687189(20)30136-7. doi: 10.1016/j.gofs.2020.03.023

5. Diagnostic and Statistical Manual of Mental Disorders (DSM- $\left.{ }^{\circledR}\right)$, Fifth Edition

American Psychiatric Association. ISBN 978-0-89042-557-2

6. [Consequences of Active Cigarette Smoking in Pregnancy - CNGOF-SFT Expert Report and Guidelines on the management of smoking during pregnancy]. Diguisto $C$, Dochez V. 
Gynecol Obstet Fertil Senol. 2020 Apr 30:S2468-7189(20)30138-0. doi: 10.1016/j.gofs.2020.03.025.

7. [In Utero Exposure to Maternal Smoking: Impact on the Child from Birth to Adulthood CNGOF-SFT Expert Report and Guidelines for Smoking Management during Pregnancy].

Torchin H, Le Lous M, Houdouin V. Gynecol Obstet Fertil Senol. 2020 Apr 1:S24687189(20)30139-2. doi: 10.1016/j.gofs.2020.03.026.

8. [Passive Smoking and Pregnancy - CNGOF-SFT Expert Report and Guidelines for Smoking Management during Pregnancy]. Rault E, Garabedian C. Gynecol Obstet Fertil Senol. 2020 Apr 1:S2468-7189(20)30140-9. doi: 10.1016/j.gofs.2020.03.027.

9. [Other Methods of Consumption: Electronic Cigarette, Heated Tobacco, Shisha and Snus CNGOF-SFT Expert Report and Guidelines on the management for Smoking Management During Pregnancy]. Garabedian C, Berveiller P, Guerby P. Gynecol Obstet Fertil Senol. 2020 Apr 1:S2468-7189(20)30144-6. doi: 10.1016/j.gofs.2020.03.031.

10 [What Policy for the Management of Smoking During Pregnancy? - CNGOF-SFT Expert Report and Guidelines on the management for Smoking Management During Pregnancy]. Koch A, Blanc J, Berlin I. Gynecol Obstet Fertil Senol. 2020 Apr 1:S2468-7189(20)30141-0. doi: $10.1016 /$ j.gofs.2020.03.028

11. [Non-pharmacological management of smoking cessation during pregnancy- CNGOF-SFT Expert Report and Guidelines for Smoking Management during Pregnancy]. Peyronnet V, Koch A, Rault E, Perdriolle-Galet E, Bertholdt C. Gynecol Obstet Fertil Senol. $2020 \mathrm{Apr}$ 2:S2468-7189(20)30142-2 doi: 10.1016/j.gofs.2020.03.029.

12. . [Pharmacotherapy for Smoking Cessation During Pregnancy - CNGOF-SFT Expert Report and Guidelines for Smoking Management During Pregnancy]. Blanc J, Koch A. Gynecol Obstet Fertil Senol. 2020 Apr 1:S2468-7189(20)30143-4. doi: 10.1016/j.gofs.2020.03.030.

13. Claire R, Chamberlain C, Davey MA, Cooper SE, Berlin I, Leonardi-Bee J, et al. Pharmacological interventions for promoting smoking cessation during pregnancy. Cochrane Database Syst Rev. 2020 Mar 4;3(3) doi: 10.1002/14651858.CD010078.pub3

14. [Smoking and Breastfeeding - CNGOF-SFT Expert Report and Guidelines on the management for Smoking Management During Pregnancy]. Le Lous M, Torchin H. Gynecol Obstet Fertil Senol. 2020 Apr 1:S2468-7189(20)30145-8. doi: 10.1016/j.gofs.2020.03.032.

15. [Management of Resumption Risk in Postpartum for Women who Quit Smoking During Pregnancy - CNGOF-SFT Expert Report and Guidelines for Smoking Management During Pregnancy]. Perdriolle-Galet E, Peyronnet V, Bertholdt C. Gynecol Obstet Fertil Senol. 2020 Apr 2:S2468-7189(20)30146-X doi: 10.1016/j.gofs.2020.03.033. 